\title{
Circulating leptin levels are not associated with cardiovascular morbidity and mortality in women with diabetes: a prospective cohort study
}

\author{
A. M. Brennan • T. Y. Li • I. Kelesidis • A. Gavrila • \\ F. B. Hu $\cdot$ C. S. Mantzoros
}

Received: 29 January 2007 / Accepted: 31 January 2007 / Published online: 20 March 2007

(C) Springer-Verlag 2007

\begin{abstract}
Aims/hypothesis Leptin, an adipocyte-secreted hormone, plays an important role in regulating neuroendocrine and immune function as well as insulin resistance and metabolism. Our objective was to examine the relationship between leptin levels and cardiovascular morbidity and overall mortality in women with type 2 diabetes.

Subjects and methods This prospective cohort study included 1,194 women with a confirmed diagnosis of type 2 diabetes, who provided a blood sample at baseline in 19891990. Participants were followed for 12 years for the development of health outcomes including cardiovascular disease (CVD) events as well as total mortality.

Results There were 218 new CVD events and 228 deaths from all causes. Cox proportional hazards analysis was used to estimate the relative risks (RRs) for each quintile level of leptin compared with the lowest quintile. Leptin levels were positively associated with several CVD risk
\end{abstract}

A. M. Brennan · I. Kelesidis • A. Gavrila • C. S. Mantzoros ( $\square)$

Division of Endocrinology, Diabetes and Metabolism,

Beth Israel Deaconess Medical Center, Harvard Medical School,

E/St 816, 330 Brookline Ave,

Boston, MA 02215, USA

e-mail: cmantzor@bidmc.harvard.edu

T. Y. Li • F. B. Hu

Department of Nutrition, Harvard School of Public Health,

Boston, MA, USA

F. B. $\mathrm{Hu}$

Department of Epidemiology,

Harvard School of Public Health,

Boston, MA, USA

F. B. $\mathrm{Hu}$

Channing Laboratory, Department of Medicine,

Brigham and Women's Hospital and Harvard Medical School,

Boston, MA, USA factors including BMI and inflammatory markers, but were not independently associated with the incidence of CVD or total mortality in women with diabetes. The multivariate RRs (95\% CIs) for CVD across the quintiles of leptin were $0.96(0.61-1.53), 0.99(0.61-1.61), 1.04$ (0.63-1.71), 1.02 $(0.59-1.75)(p$ for trend $=0.83)$.

Conclusions/interpretation Although circulating leptin levels are associated with obesity and inflammatory markers, they are not significantly related to the risk of CVD or mortality in women with diabetes.

Keywords Cardiovascular disease - Leptin - Mortality . Type 2 diabetes

$\begin{array}{ll}\text { Abbreviations } \\ \text { apo } & \text { Apolipoprotein } \\ \text { CABG } & \text { Coronary artery bypass graft } \\ \text { CRP } & \text { C-reactive protein } \\ \text { CVD } & \text { Cardiovascular disease } \\ \text { Lp(a) } & \text { Lipoprotein(a) } \\ \text { MET } & \text { Metabolic equivalent task } \\ \text { MI } & \text { Myocardial infarction } \\ \text { NHS } & \text { Nurses' Health Study } \\ \text { PTCA } & \text { Percutaneous transluminal coronary angioplasty } \\ \text { RR } & \text { Relative risk } \\ \text { sICAM-1 } & \text { Soluble intercellular adhesion molecule 1 }\end{array}$

\section{Introduction}

Leptin is an adipocyte-secreted hormone that plays a key role in neuroendocrine function, and energy homeostasis, as well as insulin resistance and metabolism. Leptin has also been shown to play an important role in regulating immune 
function in hypoleptinaemic states, as leptin administration improves immunodeficiency in both mice [1] and humans with congenital leptin deficiency [2] and improves circulating cytokine levels in subjects with relative leptin deficiency [3, 4]. Human obesity is generally associated with a state of hyperleptinaemia and leptin resistance, and leptin levels are elevated in proportion to the degree of adiposity in the vast majority of obese humans [5]. In addition, in cross-sectional studies leptin levels have been associated with insulin resistance and the proinflammatory state that accompanies obesity, but whether this association is causal or due to an underlying association with confounding variables remains to be clarified.

While some observational studies have shown that leptin is associated with inflammatory markers independently of fat mass [6-8], others have demonstrated that this relationship becomes non-significant with careful control for measures of body fat [9]. Similarly, although interventional studies in humans have shown that leptin administration in low leptin states is associated with increased inflammatory and platelet responses [3, 10], there appears to be no significant effect of leptin administration in leptin-replete states such as obesity [11].

Mainly on the basis of the reported associations with inflammatory markers and fat mass, it has been suggested that elevated levels of leptin may be an independent risk factor for cardiovascular disease (CVD). Observational studies have mainly focused on men and have yielded conflicting results regarding the role of elevated leptin levels as a risk factor for new cardiovascular events, with some studies supporting an association [12-15], others reporting no association independent of body mass [16] and a further study, in women with type 2 diabetes, reporting that leptin had a protective effect on cardiovascular mortality [17]. These conflicting results may be due to limited power and/ or limited duration of follow-up. They may also be due to inadequate control for confounding variables, since no prior study has performed a joint evaluation of all CVD risk factors including $\mathrm{HbA}_{1 \mathrm{c}}$ and inflammatory biomarkers.

To examine prospectively the association between leptin and cardiovascular morbidity and mortality as well as allcause mortality in women with diabetes, we examined the associations between leptin levels and cardiovascular events in a large cohort of 1,194 women with diabetes, followed for 12 years in the context of the prospective Nurses' Health Study (NHS) before and after controlling for the potential confounding effects of multiple cardiovascular risk factors.

\section{Subjects and methods}

Study population The NHS was initiated in 1976 with the enrolment of 121,700 US nurses, aged $30-55$ years. This prospective cohort study involves biannually mailed questionnaires related to lifestyle factors and health outcomes. In 1989-1990, 32,826 study participants provided blood samples by overnight courier. The present study includes 1,194 of those women who had a confirmed diagnosis of type 2 diabetes after excluding women with prevalent or incident malignancy [18]; of those, 957 did not report prior diagnosis of myocardial infarction (MI), coronary revascularisation or stroke at the time of blood drawing. The study was approved by the Human Research Committees at the Brigham and Women's Hospital and the Beth Israel Deaconess Medical Center; completion of the self-administered questionnaire was considered to imply informed consent.

Definition of diabetes, CHD and CVD The respondents reported cases of diabetes on the biannual questionnaires. A supplementary questionnaire was mailed to all women reporting a diagnosis of diabetes to obtain additional information about the date of diagnosis, symptoms, diagnostic tests and treatment. A follow-up questionnaire confirmed a diagnosis of diabetes in $98 \%$ of women. We used the National Diabetes Data Group criteria to define diabetes because all our subjects were diagnosed before the American Diabetes Association released their criteria in 1997. The validity of self-reported diagnosis of type 2 diabetes by our supplementary questionnaire has been established by a separate and independent validation study through medical record reviews [19].

CVD endpoints consist of fatal MI, MI, coronary artery bypass graft (CABG), percutaneous transluminal coronary angioplasty (PTCA) and stroke. CHD endpoints consist of fatal MI, MI, CABG and PTCA. Self-reported MIs and strokes were confirmed by medical record review as previously described $[20,21]$. Deaths were reported to the NHS group by the subjects' next of kin, work associates and postal authorities; in some cases, the National Death Index of the National Center for Health Statistics was used to identify deceased cohort members. Fatal CHD was confirmed by a review of medical records or autopsy reports with the permission of the next of kin [21].

Blood collection and processing Blood was drawn in 1989-1990. Participants were sent a blood-set kit that included supplies (blood tubes, tourniquet, needles, bandage and coolant pack) and instructions. Participants arranged for the blood to be drawn and sent the samples back by prepaid overnight courier. Most samples arrived within $24 \mathrm{~h}$ of the blood drawing. After arrival in the laboratory, samples were centrifuged and aliquoted into cryotubes as plasma, buffy coat and erythrocytes. Cryotubes were stored in liquid-nitrogen freezers at $-130^{\circ} \mathrm{C}$ or lower. Leptin was assayed by RIA as previously described 
[22] (Linco Research, Inc., St Charles, MO, USA; sensitivity $0.5 \mathrm{ng} / \mathrm{ml}$; intra-assay $\mathrm{CV} 3.4-8.3 \%$ ). Leptin has been previously shown to be a stable analyte [23]. TNF- $\alpha$ was assayed using a commercially available ELISA (Quantikine HS; R\&D Systems, Minneapolis, MN, USA; sensitivity $3 \mathrm{pg} / \mathrm{ml}$; CV 2.7-6.9\%). Plasma C-reactive protein (CRP) was measured using a US CRP ELISA kit as previously described [18] (Diagnostic Systems Laboratories, Inc., Webster, TX, USA; CV 2.8-5.1\%) Soluble intercellular adhesion molecule (sICAM-1) was assayed in plasma using a human sICAM-1 ELISA kit (R\&D Systems; CV 3.3-4.8\%). Plasma levels of sE-selectin were assayed using the human sE-selectin ELISA kit (R\&D Systems; CV 5.7-8.8\%). Concentrations of $\mathrm{HbA}_{1 \mathrm{c}}$ were based on turbidimetric immunoinhibition with haemolysed whole blood or packed erythrocytes with a CV $<3.0 \%$. Fibrinogen was measured on an Hitachi 911 Analyzer (Tokyo, Japan) using reagents and calibrators from Kamiya Biomedical Co. (Seattle, WA, USA) with a CV of $1.16 \%$. Adiponectin was assayed by RIA (Linco Research; sensitivity $2 \mu \mathrm{g} / \mathrm{ml}$; CV 1.78-6.21\%). The concentrations of total cholesterol, cholesterol and triacylglycerol were measured simultaneously on an Hitachi 911 Analyzer with reagents and calibrators from Roche (Indianapolis, IN, USA) in 2003; the CVs for these measurements were $<1.8 \%$. Concentrations of LDL cholesterol were measured using a homogeneous direct method from Genzyme (Cambridge, MA, USA) with a CV $<3.1 \%$. Concentrations of apolipoprotein (apo) B100 were measured in an immunonephelometric assay using reagents and calibrators from Wako Chemicals (Richmond, VA, USA) with a $\mathrm{CV}<5 \%$. Lipoprotein(a) $[\mathrm{Lp}(\mathrm{a})]$ was measured by a latex-enhanced immunoturbidimetric method as previously described [20] (Denka Sieken, Tokyo, Japan; CV 2.6\%).

Assessment of lifestyle exposures We calculated BMI as the ratio of weight $(\mathrm{kg})$ to the height $\left(\mathrm{m}^{2}\right)$. Physical activity was computed as metabolic equivalent tasks (METs) per week from data collected during the period 1986-2000 on duration and intensity of exercise [24]. History of hypertension and family history of MI were determined from self-reports before blood collection [25]. Alcohol intake was estimated with a dietary questionnaire administered in 1990, 1994 and 1998 [25].

Statistical analysis Spearman correlations and scatter plots were used to evaluate bivariate relationships between plasma levels of leptin and levels of lipoproteins or inflammatory markers at baseline (1990) before and after adjusting for BMI. Women were followed from June 1990 through to June 2002. Cox proportional hazards analysis was used to estimate the relative risks (RRs) for each quintile of leptin levels compared with the lowest quintiles after stratification on 5-year age categories and each 2-year follow-up interval. Accumulation of person-months of follow-up started in June 1990. Participants in whom CHD or stroke was diagnosed or who died during followup were censored at the date of diagnosis or death. All other participants were followed through to June 2002. We adjusted for known risk factors for cardiovascular and total morbidity and mortality such as age, BMI, physical activity (tertiles), smoking (never, past and current), aspirin use, family history of CVD, history of hypertension, history of hypercholesterolaemia, baseline fasting status, alcohol intake $(0.0,0.1-4.9,5.0-14.9$ and $15.0 \mathrm{~g} /$ day), insulin use and postmenopausal hormone replacement use as indicated in the respective tables. In separate models, we also adjusted for $\mathrm{HbA}_{1 \mathrm{c}}$, CRP, non-HDL-cholesterol and fibrinogen. Tests of linear trend across increasing categories of leptin levels were conducted by treating the categories as a continuous variable and assigning the median intake for the category as its value. For CVD and CHD analyses, women with CVD at baseline were excluded. Mortality analyses included women with baseline CVD, and additional adjustment for baseline CVD was performed. We conducted secondary analysis by classifying leptin levels by $>5$ and $\leq 5 \mathrm{ng} / \mathrm{ml},>4$ and $\leq 4 \mathrm{ng} / \mathrm{ml}$ groups, and then examining the RRs for $\leq 5 \mathrm{vs}>5 \mathrm{ng} / \mathrm{ml}$ and $\leq 4 \mathrm{vs}>4 \mathrm{ng} / \mathrm{ml}$ groups, respectively. All statistical analyses were performed with SAS 9.0 statistical software (SAS Institute, Cary, NC, USA). Due to multiple outcomes, statistical significance was set at the $p=0.01$ level.

\section{Results}

During the 12-year follow-up, we documented 218 new CVD events (including cases of fatal MI, MI, stroke, CABG and PTCA), of which 168 were CHD events. There were 103 CVD deaths and 228 deaths from all causes among 1,194 women with type 2 diabetes, including those with baseline CVD. Table 1 shows age-adjusted characteristics of risk factors, lipoproteins or inflammatory markers across the quintiles of leptin levels. Higher BMI, waist circumference, WHR and hypertension were associated with higher leptin levels. Women with lower leptin levels were more likely to smoke, use insulin and use oestrogen. Leptin levels were strongly positively associated with TNF- $\alpha$, sE-selectin, CRP, apoB ${ }_{100}$, creatinine and fibrinogen, whereas adiponectin levels decreased between the first and second quintiles but remained at similar levels thereafter.

To examine inter-relationships between biomarkers, after controlling for BMI, which could be confounding the reported associations, we calculated Spearman partial correlation coefficients (Table 2). Leptin levels were significantly associated with TNF- $\alpha$, creatinine, $\mathrm{HbA}_{1 \mathrm{c}}$, 
Table 1 Age-adjusted characteristics of risk factors, lipoprotein and biomarkers across the quintiles of leptin levels

\begin{tabular}{|c|c|c|c|c|c|c|}
\hline & \multicolumn{5}{|l|}{ Quintile } & \multirow[t]{2}{*}{$p$ value } \\
\hline & $1(n=195)$ & $2(n=201)$ & $3(n=188)$ & $4(n=194)$ & $5(n=179)$ & \\
\hline \multicolumn{7}{|l|}{ Leptin level (ng/ml) } \\
\hline Range & $0-21.42$ & $21.43-37.39$ & $37.40-53.28$ & $53.28-70.02$ & $>70.02$ & \\
\hline Mean & 12.22 & 29.00 & 45.52 & 60.81 & 91.74 & $<0.001$ \\
\hline \multicolumn{7}{|l|}{ Descriptive variables (means) } \\
\hline Age (years) & 58.4 & 58.5 & 58.3 & 58.4 & 58.4 & 0.45 \\
\hline BMI $\left(\mathrm{kg} / \mathrm{m}^{2}\right)$ & 23.8 & 27.6 & 30.6 & 32.2 & 36.5 & $<0.001$ \\
\hline Waist circumference $(\mathrm{cm})$ & 79.8 & 88.1 & 93.5 & 97.0 & 100.6 & $<0.001$ \\
\hline WHR & 0.81 & 0.84 & 0.87 & 0.86 & 0.84 & 0.007 \\
\hline Alcohol consumption (g/day) & 3.01 & 3.44 & 2.85 & 2.61 & 1.93 & 0.33 \\
\hline Physical activity (METs/week) & 39.6 & 31.6 & 34.9 & 58.0 & 48.4 & 0.27 \\
\hline Current smoker (\%) & 21.2 & 16.8 & 15.5 & 6.8 & 8.0 & $<0.001$ \\
\hline Never smokers $(\%)$ & 41.6 & 42.3 & 50.8 & 47.1 & 47.1 & 0.36 \\
\hline Aspirin use (\%) & 60.9 & 57.8 & 57.7 & 58.4 & 55.2 & 0.84 \\
\hline Postmenopausal status + hormone use $(\%)$ & 42.3 & 38.7 & 34.1 & 30.1 & 23.6 & 0.01 \\
\hline Family history of MI (\%) & 24.1 & 26.1 & 18.7 & 21.4 & 28.2 & 0.25 \\
\hline Hypertension (\%) & 30.6 & 35.1 & 44.2 & 53.1 & 53.2 & $<0.001$ \\
\hline Hypercholesterolaemia (\%) & 36.9 & 41.8 & 44.9 & 46.2 & 34.2 & 0.12 \\
\hline Insulin medication (\%) & 27 & 14 & 16 & 17 & 21 & 0.01 \\
\hline \multicolumn{7}{|l|}{ Biomarkers (means) } \\
\hline sICAM-1 (ng/ml) & 300.1 & 308.4 & 318.9 & 313.6 & 329.2 & 0.08 \\
\hline $\mathrm{TNF}-\alpha(\mathrm{pg} / \mathrm{ml})$ & 2,298 & 2,320 & 2,513 & 2,704 & 2,975 & $<0.001$ \\
\hline sE-selectin (ng/ml) & 53.9 & 64.7 & 70.7 & 67.7 & 74.5 & $<0.001$ \\
\hline $\mathrm{CRP}(\mathrm{mg} / \mathrm{l})$ & 4.41 & 7.51 & 8.37 & 9.89 & 11.44 & $<0.001$ \\
\hline Adiponectin $(\mu \mathrm{g} / \mathrm{ml})$ & 10.72 & 6.85 & 6.77 & 6.14 & 6.58 & $<0.001$ \\
\hline $\mathrm{HbA}_{1 \mathrm{c}}(\%)$ & 7.08 & 6.95 & 7.12 & 6.57 & 6.61 & 0.002 \\
\hline apoB100 (g/l) & 0.96 & 10.36 & 10.61 & 10.45 & 10.29 & $<0.001$ \\
\hline Total cholesterol (mmol/l) & 5.67 & 5.83 & 5.93 & 5.85 & 5.83 & 0.17 \\
\hline Creatinine $(\mu \mathrm{mol} / \mathrm{l})$ & 67.8 & 65.12 & 66.88 & 69.50 & 73.90 & $<0.001$ \\
\hline Fibrinogen (mmol/l) & 10.17 & 10.88 & 11.08 & 11.39 & 11.82 & $<0.001$ \\
\hline HDL-cholesterol (mmol/l) & 1.4 & 1.29 & 1.29 & 1.27 & 1.29 & $<0.001$ \\
\hline LDL-cholesterol (mmol/l) & 3.41 & 3.50 & 3.73 & 3.40 & 3.62 & 0.02 \\
\hline $\mathrm{LP}(\mathrm{a})(\mu \mathrm{mol} / \mathrm{l})$ & 0.71 & 0.60 & 0.66 & 0.60 & 0.67 & 0.67 \\
\hline Fasting triacylglycerol $(\mathrm{mmol} /)^{\mathrm{a}}$ & 2.08 & 2.51 & 2.35 & 2.29 & 2.09 & 0.29 \\
\hline Non-HDL-cholesterol (mmol/l) & 4.23 & 4.53 & 4.64 & 4.59 & 4.54 & 0.003 \\
\hline Total cholesterol:HDL-cholesterol ratio & 4.31 & 4.94 & 4.88 & 4.92 & 4.76 & $<0.001$ \\
\hline
\end{tabular}

${ }^{\text {a }}$ Measures of fasting triacylglycerol available for $n=639$ subjects

Baseline exclusions: fatal MI, MI, CABG and stroke

CRP and fibrinogen and weakly associated with sE-selectin and sICAM-1. The association between leptin and lipoproteins was non-significant after controlling for BMI.

Importantly, we did not observe a significant association between quintiles of leptin levels and incidence of CHD or CVD in women with diabetes (Table 3). The multivariate RRs (95\% CIs) for CVD across the quintiles of leptin levels were 0.96 (0.61-1.53), 0.99 (0.61-1.61), 1.04 (0.63-1.71) and $1.02(0.59-1.75)$, respectively ( $p$ for trend $=0.83)$. The associations were not changed appreciably after additional adjustment for insulin treatment, CRP, non-HDL-cholesterol, fibrinogen and $\mathrm{HbA}_{1 \mathrm{c}}$. Moreover, the multivariate $\mathrm{RR}$ of continuous leptin levels with CVD events was 1.00 (0.991.01). Additional adjustment for time from blood draw to analysis and limitation of analysis to subjects with fasting samples only did not alter the result.

In addition, we did not find any significant association between quintiles of leptin level and cardiovascular mortality or all-cause mortality on univariate analysis. Multivariate analysis, including anthropometric, demographic and lifestyle factors revealed a significant association for all-cause mortality and an association approaching significance for CVD mortality; however, following adjustment for glycaemic control and inflammatory biomarkers, the association was non-significant (Table 4).

Finally, to explore whether relative hypoleptinaemia is associated with mortality and morbidity, we used previously defined cut-off levels for hypoleptinaemia to evaluate the 


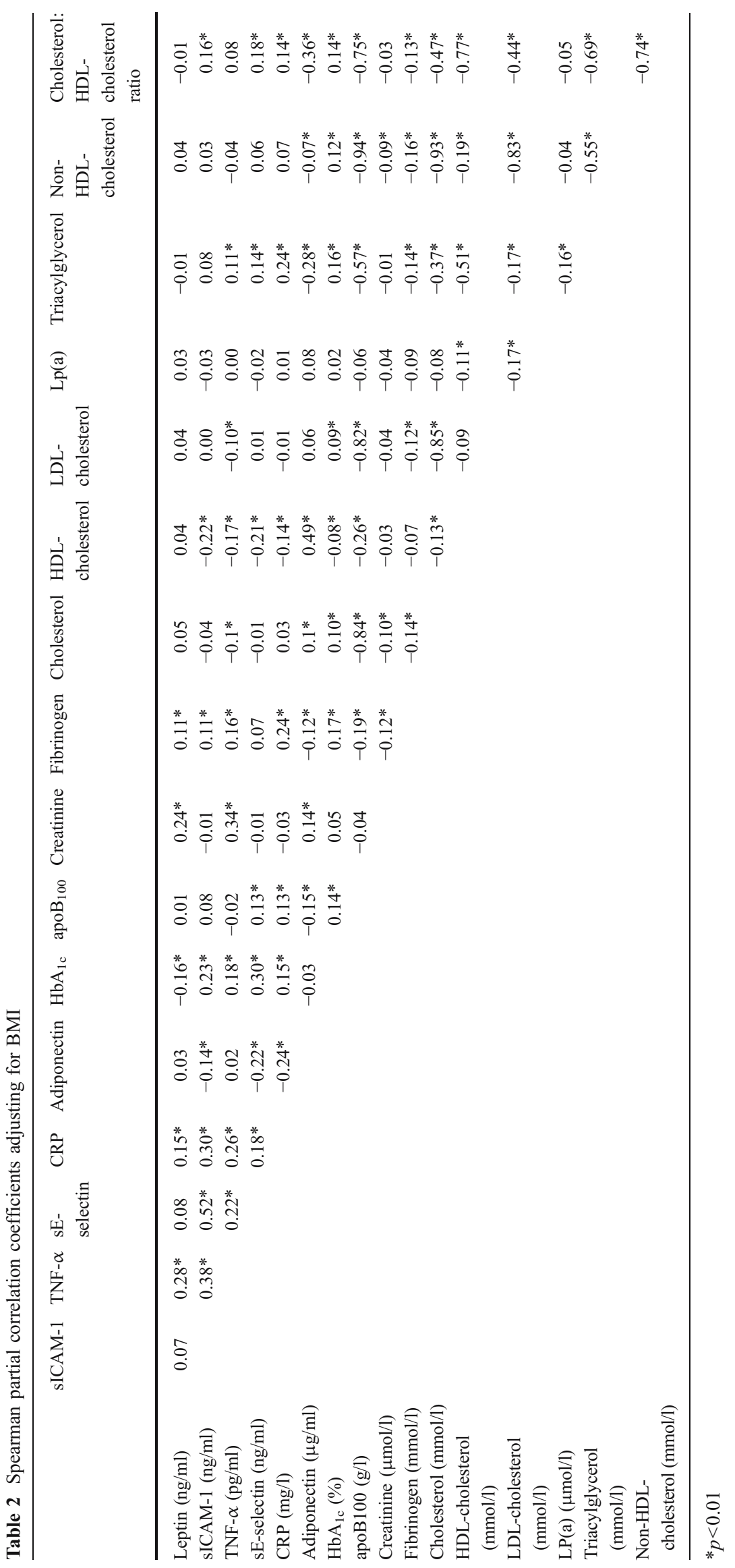


Table 3 CVD and CHD in relation to quintiles of leptin levels (RR and 95\% CI) for women without baseline CVD

\begin{tabular}{|c|c|c|c|c|c|c|}
\hline & \multicolumn{5}{|c|}{ Quintile (ng/ml leptin) } & \multirow[t]{2}{*}{$p$ value for trend } \\
\hline & $1(<21.42)$ & $2(21.43-37.39)$ & $3(37.45-0.28)$ & $4(53.29-70.02)$ & $5(>70.02)$ & \\
\hline Person-years & 2,006 & 2,101 & 1,927 & 1,917 & 1,836 & \\
\hline CVD cases & 39 & 44 & 44 & 50 & 41 & \\
\hline Age-adjusted & 1.0 (ref) & $1.06(0.69-1.64)$ & $1.17(0.76-1.80)$ & $1.31(0.86-1.99)$ & $1.16(0.75-1.80)$ & 0.26 \\
\hline MV & 1.0 (ref) & $0.96(0.61-1.53)$ & $0.99(0.61-1.61)$ & $1.04(0.63-1.71)$ & $1.02(0.59-1.75)$ & 0.83 \\
\hline $\begin{array}{l}\mathrm{MV}+\mathrm{CRP}, \text { non-HDL, } \\
\text { fibrinogen, } \mathrm{HbA}_{1 \mathrm{c}}\end{array}$ & 1.0 (ref) & $1.01(0.63-1.60)$ & $1.06(0.65-1.71)$ & $1.20(0.72-1.98)$ & $1.17(0.67-2.03)$ & 0.42 \\
\hline CHD cases & 30 & 36 & 30 & 43 & 29 & \\
\hline Age-adjusted & 1.0 (ref) & $1.14(0.70-1.85)$ & $1.04(0.63-1.72)$ & $1.46(0.91-2.32)$ & $1.08(0.65-1.80)$ & 0.38 \\
\hline MV & 1.0 (ref) & $0.99(0.57-1.67)$ & $0.87(0.50-1.54)$ & $1.09(0.63-1.90)$ & $0.88(0.47-1.64)$ & 0.92 \\
\hline $\begin{array}{l}\mathrm{MV}+\mathrm{CRP} \text {, non-HDL-cholesterol, } \\
\text { fibrinogen, } \mathrm{HbA}_{1 \mathrm{c}}\end{array}$ & 1.0 (ref) & $1.04(0.62-1.76)$ & $0.94(0.53-1.85)$ & $1.29(0.73-2.25)$ & $1.05(0.55-1.99)$ & 0.58 \\
\hline
\end{tabular}

$M V$, Adjusted for age, BMI, physical activity (three categories), smoking (never, past and current), aspirin use, family history of CVD, baseline hypertension, baseline hypercholesterolaemia, fasting status, alcohol intake (0.0, 0.1-4.9, 5.0-14.9 and $15.0 \mathrm{~g} /$ day), insulin treatment and postmenopausal hormone replacement use

clinical cut-off point of protective leptin levels for incidence of CVD. The group with leptin levels $\leq 5 \mathrm{ng} / \mathrm{ml}$ did not have a higher risk of CVD compared with those with leptin level $>5 \mathrm{ng} / \mathrm{ml}$. The multivariate RRs of leptin $\leq 4 \mathrm{ng} / \mathrm{ml}$ was 1.27 (0.50-3.25) compared with leptin levels $>4 \mathrm{ng} / \mathrm{ml}$ and leptin $\leq 5 \mathrm{ng} / \mathrm{ml}$ was $1.67(0.77-3.62)$ compared with leptin levels $>5 \mathrm{ng} / \mathrm{ml}$.

\section{Discussion}

We confirm herein that leptin levels are associated with adiposity as well as several cardiovascular risk factors [6-8], but despite these associations, we failed to detect any independent significant association between leptin and either cardiovascular morbidity and/or mortality in women with type 2 diabetes.
Previous observational studies on the association between elevated leptin and CVD have reported conflicting results [12-15]. A case-control study nested in the West of Scotland Coronary Prevention Study found elevated leptin levels to be a predictor of CVD events in men, but the authors did not control for all potential confounders and leptin retained only a borderline significant association after adjustment for CRP [12]. In another study, plasma leptin levels have been associated with first-ever MI in men [13] and haemorrhagic but not ischaemic stroke [14] in men and women. Neither of these studies adjusted for markers of inflammation, which are known to be associated with both high leptin levels and CVD, and a subsequent larger casereferent study from the same population refuted the above findings by reporting that leptin independently predicted stroke in men but not women after adjusting for CRP [15]. The confounding effect of inflammatory biomarkers is

Table 4 Mortality and quintiles of leptin levels (RR and 95\% CI)

\begin{tabular}{|c|c|c|c|c|c|c|}
\hline & \multicolumn{5}{|c|}{ Quintile (ng/ml leptin) } & \multirow[t]{2}{*}{$p$ for trend } \\
\hline & $1(<21.42)$ & $2(21.43-37.39)$ & $3(37.4-53.28)$ & $4(53.29-70.02)$ & $5(>70.02)$ & \\
\hline Person years & 2,614 & 2,713 & 2,664 & 2,685 & 2,615 & \\
\hline CVD mortality $(n)$ & 19 & 16 & 18 & 20 & 30 & \\
\hline Age-adjusted & 1.0 (ref) & $0.78(0.40-1.53)$ & $0.88(0.46-1.69)$ & $0.96(0.51-1.81)$ & $1.58(0.89-2.81)$ & 0.10 \\
\hline $\begin{array}{l}\mathrm{MV}+\mathrm{TNF}-\alpha \text {, creatinine, } \mathrm{HbA}_{1 \mathrm{c}} \text {, CRP, } \\
\text { fibrinogen, sE-selectin, sICAM- } 1^{\mathrm{a}}\end{array}$ & 1.0 (ref) & $0.82(0.39-1.72)$ & $1.05(0.50-2.20)$ & $0.90(0.42-1.95)$ & $1.20(0.55-2.64)$ & 0.63 \\
\hline All-cause mortality $(n)$ & 45 & 36 & 41 & 48 & 58 & \\
\hline Age-adjusted & 1.0 (ref) & $0.75(0.48-1.16)$ & $0.86(0.57-1.32)$ & $0.99(0.66-1.48)$ & $1.31(0.89-1.93)$ & 0.10 \\
\hline $\begin{array}{l}\mathrm{MV}+\mathrm{TNF}-\alpha, \text { creatinine, } \mathrm{HbA}_{1 \mathrm{c}} \text {, CRP, } \\
\text { fibrinogen, sE-selectin, sICAM- }{ }^{\mathrm{a}}\end{array}$ & 1.0 (ref) & $0.75(0.45-1.23)$ & $1.02(0.62-1.67)$ & $1.07(0.64-1.80)$ & $1.00(0.58-1.72)$ & 0.54 \\
\hline
\end{tabular}

$M V$, adjusted for age, BMI, physical activity (three categories), smoking (never, past and current), aspirin use, family history of CVD, baseline hypertension, baseline hypercholesterolaemia, fasting status, alcohol intake (0.0, 0.1-4.9, 5.0-14.9 and $15.0 \mathrm{~g} /$ day), insulin treatment and postmenopausal hormone replacement use

${ }^{a}$ Additional adjustment for prior diagnosis of CVD at baseline did not alter these results 
confirmed by the current study, which shows that adjusting for anthropometric, demographic and lifestyle factors only there appears to be a trend towards a weak relationship between quintiles of leptin levels and all-cause mortality. This association is clearly non-significant when glycaemic control and inflammatory biomarkers are included in the analysis as potential confounders.

Further, although the lack of association with CVD events has been previously reported in a prospective study of heart disease in French-Canadian men [16], this study followed subjects for only 5 years, included soft cardiovascular outcomes such as effort angina and did not include inflammatory biomarkers. Endogenous circulating leptin levels are higher in women than men for a given fat mass, but whether this reflects different signalling remains unknown given that no studies directly assessing leptin signalling have been performed in humans, either men or women. The role of leptin as an independent risk factor for CVD has not yet been studied in women with diabetes, but a previous cohort study has examined the association with cardiovascular mortality in middle-aged glucose-intolerant women [17]. In this study, leptin levels in the lowest tertile were negatively and independently associated with cardiovascular mortality in both univariate and multivariate analyses [17], including age, serum insulin levels, BMI and monocyte chemoattracting protein, but not other traditional risk factors for CVD. The suggestion of a protective effect of elevated serum leptin levels, in that a preliminary small study ( $n=17$ cases only), together with results from two recent human interventional studies in individuals with relative leptin deficiency and congenital lipoatrophy [26, 27], as well as HIV lipoatrophy and the metabolic syndrome [28], showing that leptin-deficient subjects have reduced insulin sensitivity that is improved with leptin administration, prompted us to examine more closely the risk of CVD in women by stratifying for leptin levels below the previously used clinical cut-off levels for hypoleptinaemia [4]. We observed no significantly increased risk of CVD in women with leptin levels $<4 \mathrm{ng} / \mathrm{ml}$ or $<5 \mathrm{ng} / \mathrm{ml}$ before and/or after adjustment for confounders. Although similar results were observed when, instead of a cut-off point we used the lowest quintile, the possibility exists that a much larger sample size may be able to detect increased risk as the subgroup of women with leptin levels $<5 \mathrm{ng} / \mathrm{ml}$ represented only a relatively small proportion $(<5 \%)$ of the overall sample in this study, as it does in the general population.

A limitation of the current study is our inability to address causality. Cohort studies are not affected by recall or selection bias and incorporate the time sequence criterion for causality and we have adjusted for known confounders but only large randomised clinical trials can prove causality. Also, whether leptin stimulates inflammatory biomarkers and thereby increases the risk of CVD in high leptin states such as obesity, remains unknown. We have previously performed interventional studies in normo- or hyperleptinaemic lean and obese men and women, demonstrating that increasing leptin levels, through leptin administration, did not result in any significant change in several inflammatory markers associated with CVD in both short-term (3 days) and longer-term (16 weeks) studies [11]. In contrast, in human studies involving leptin administration in low leptin states, including congenital leptin deficiency and starvationinduced hypoleptinaemia, leptin administration was associated with a significant increase in inflammatory markers and increased platelet aggregation $[3,10]$. On the basis of the differing biological activity of leptin in low vs high leptin states the association with inflammatory biomarkers in obesity, a hyperleptinaemic leptin-resistant state, does not appear to be causal. Further studies are required to confirm the findings from our interventional studies in leptin-replete states [11].

In summary, we failed to detect any independent association between serum leptin levels and CHD, CVD, cardiovascular mortality and all-cause mortality in women with diabetes. This study has several strengths including its prospective design, the large sample size and prolonged follow-up that increase its power, and the blinded evaluation of the serum analytes. Since the observational nature precludes any conclusion on causality, interventional studies are required to further investigate the relationship between leptin, inflammatory biomarkers and CVD.

Acknowledgements This work was supported by NIH Grant DK58785, a BIDMC discretionary grant and Tanita grant-in-aid to C. S. Mantzoros and grants HL-65582, DK-58845 and American Heart Association Established Investigator Award to F. B. Hu.

Duality of interest The authors declare that they have no duality of interest.

\section{References}

1. Howard JK, Lord GM, Matarese G et al (1999) Leptin protects mice from starvation-induced lymphoid atrophy and increases thymic cellularity in ob/ob mice. J Clin Invest 104:1051-1059

2. Farooqi IS, Matarese G, Lord GM et al (2002) Beneficial effects of leptin on obesity, T cell hyporesponsiveness, and neuroendocrine/metabolic dysfunction of human congenital leptin deficiency. J Clin Invest 110:1093-1103

3. Chan JL, Moschos SJ, Bullen J et al (2005) Recombinant methionyl human leptin administration activates signal transducer and activator of transcription 3 signaling in peripheral blood mononuclear cells in vivo and regulates soluble tumor necrosis factor-alpha receptor levels in humans with relative leptin deficiency. J Clin Endocrinol Metab 90:1625-1631

4. Welt CK, Chan JL, Bullen J et al (2004) Recombinant human leptin in women with hypothalamic amenorrhea. N Engl J Med 351:987-997 
5. Considine RV, Sinha MK, Heiman ML et al (1996) Serum immunoreactive-leptin concentrations in normal-weight and obese humans. N Engl J Med 334:292-295

6. Shamsuzzaman AS, Winnicki M, Wolk R et al (2004) Independent association between plasma leptin and $\mathrm{C}$-reactive protein in healthy humans. Circulation 109:2181-2185

7. van Dielen FM, van't Veer C, Schols AM, Soeters PB, Buurman WA, Greve JW (2001) Increased leptin concentrations correlate with increased concentrations of inflammatory markers in morbidly obese individuals. Int J Obes Relat Metab Disord 25:1759-1766

8. Gomez-Ambrosi J, Salvador J, Paramo JA et al (2002) Involvement of leptin in the association between percentage of body fat and cardiovascular risk factors. Clin Biochem 35:315-320

9. Gomez-Ambrosi J, Salvador J, Silva C et al (2005) Leptin therapy does not affect inflammatory markers. J Clin Endocrinol Metab 90:3803

10. Canavan B, Salem RO, Schurgin S et al (2005) Effects of physiological leptin administration on markers of inflammation, platelet activation, and platelet aggregation during caloric deprivation. J Clin Endocrinol Metab 90:5779-5785

11. Chan JL, Bullen J, Stoyneva V, DePaoli AM, Addy C, Mantzoros CS (2005) Recombinant methionyl human leptin administration to achieve high physiologic or pharmacologic leptin levels does not alter circulating inflammatory marker levels in humans with leptin sufficiency or excess. J Clin Endocrinol Metab 90:1618-1624

12. Wallace AM, McMahon AD, Packard CJ et al (2001) Plasma leptin and the risk of cardiovascular disease in the West of Scotland Coronary Prevention Study (WOSCOPS). Circulation 104:3052-3056

13. Soderberg S, Ahren B, Jansson JH et al (1999) Leptin is associated with increased risk of myocardial infarction. J Intern Med 246:409-418

14. Soderberg S, Ahren B, Stegmayr B et al (1999) Leptin is a risk marker for first-ever hemorrhagic stroke in a population-based cohort. Stroke 30:328-337

15. Soderberg S, Stegmayr B, Stenlund H et al (2004) Leptin, but not adiponectin, predicts stroke in males. J Intern Med 256:128-136

16. Couillard C, Lamarche B, Mauriege P et al (1998) Leptinemia is not a risk factor for ischemic heart disease in men. Prospective results from the Quebec Cardiovascular Study. Diabetes Care 21:782-786

17. Piemonti L, Calori G, Mercalli A et al (2003) Fasting plasma leptin, tumor necrosis factor-alpha receptor 2, and monocyte chemoattracting protein 1 concentration in a population of glucose-tolerant and glucose-intolerant women: impact on cardiovascular mortality. Diabetes Care 26:2883-2889

18. Mantzoros CS, Li T, Manson JE, Meigs JB, Hu FB (2005) Circulating adiponectin levels are associated with better glycemic control, more favorable lipid profile, and reduced inflammation in women with type 2 diabetes. J Clin Endocrinol Metab 90:45424548

19. Manson JE, Colditz GA, Stampfer MJ et al (1991) A prospective study of maturity-onset diabetes mellitus and risk of coronary heart disease and stroke in women. Arch Intern Med 151:11411147

20. Shai I, Schulze MB, Manson JE, Stampfer MJ, Rifai N, Hu FB (2005) A prospective study of lipoprotein(a) and risk of coronary heart disease among women with type 2 diabetes. Diabetologia 48:1469-1476

21. Tanasescu M, Cho E, Manson JE, Hu FB (2004) Dietary fat and cholesterol and the risk of cardiovascular disease among women with type 2 diabetes. Am J Clin Nutr 79:999-1005

22. Gavrila A, Chan JL, Yiannakouris N et al (2003) Serum adiponectin levels are inversely associated with overall and central fat distribution but are not directly regulated by acute fasting or leptin administration in humans: cross-sectional and interventional studies. J Clin Endocrinol Metab 88:4823-4831

23. Ma Z, Gingerich RL, Santiago JV, Klein S, Smith CH, Landt M (1996) Radioimmunoassay of leptin in human plasma. Clin Chem 42:942-946

24. Hu FB, Meigs JB, Li TY, Rifai N, Manson JE (2004) Inflammatory markers and risk of developing type 2 diabetes in women. Diabetes 53:693-700

25. Shai I, Rosner BA, Shahar DR et al (2005) Dietary evaluation and attenuation of relative risk: multiple comparisons between blood and urinary biomarkers, food frequency, and 24-hour recall questionnaires: the DEARR study. J Nutr 135:573-579

26. Oral EA, Simha V, Ruiz E et al (2002) Leptin-replacement therapy for lipodystrophy. N Engl J Med 346:570-578

27. Javor ED, Cochran EK, Musso C, Young JR, DePaoli AM, Gorden P (2005) Long-term efficacy of leptin replacement in patients with generalized lipodystrophy. Diabetes 54:1994-2002

28. Lee JH, Chan JL, Sourlas E, Raptopoulos V, Mantzoros CS (2006) Recombinant methionyl human leptin therapy in replacement doses improves insulin resistance and metabolic profile in patients with lipoatrophy and metabolic syndrome induced by the highly active antiretroviral therapy. J Clin Endocrinol Metab 91:2605-2611 